\title{
Evolving theoretical descriptions of heavy-ion fusion: from phenomenologi- cal to microscopic approaches
}

\author{
K. Hagino ${ }^{1,2,3}$ \\ ${ }^{1}$ Department of Physics, Tohoku University, Sendai 980-8578, Japan \\ ${ }^{2}$ Research Center for Electron Photon Science, Tohoku University, 1-2-1 Mikamine, Sendai 982-0826, Japan \\ ${ }^{3}$ National Astronomical Observatory of Japan, 2-21-1 Osawa, Mitaka, Tokyo 181-8588, Japan
}

\begin{abstract}
We overview the current status of theoretical approaches for heavy-ion fusion reactions at subbarrier energies. We particularly discuss theoretical challenges in the coupled-channels approach, that include i) a description of deep subbarrier hindrance of fusion cross sections, ii) the role of nuclear dissipation, iii) fusion of unstable nuclei, and iv) an interplay between fusion and multi-nucleon transfer processes. We also present results of a semi-microscopic approach to heavy-ion fusion reactions, that combines the coupled-channels approach with state-of-the-art microscopic nuclear structure calculations.
\end{abstract}

\section{Introduction}

Fusion is a reaction to form a compound nucleus. It plays an important role in several phenomena in nuclear physics and nuclear astrophysics, such as synthesis of superheavy elements and the energy production in stars. In the potential between two colliding nuclei, the so called Coulomb barrier is formed by a strong cancellation between the Coulomb and a nuclear interactions. This Coulomb barrier has to be overcome in order for fusion to take place, and thus the height of the Coulomb barrier defines the energy scale of the reaction. In this contribution, we mainly consider fusion reactions in heavy-ion systems at energies around the Coulomb barrier, that is, heavy-ion subbarrier fusion reactions.

Why are we interested in subbarrier fusion reactions? One obvious reason for this is that subbarrier fusion is relevant to superheavy elements and nuclear astrophysics. That is, many superheavy elements have been synthesized by heavy-ion fusion reactions at energies slightly above the Coulomb barrier. Also, it is crucially important to understand the dynamics of subbarrier fusion reactions in order to make a reliable extrapolation of experimental data down to extremely low energies at which fusion reaction takes place in stars.

Besides this obvious reason, there are many other good reasons why subbarrier fusion reactions are interesting to study. Firstly, there is a strong interplay between the nuclear reaction and the nuclear structure in there. This strong interplay has been well realized in a large enhancement of fusion cross sections at subbarrier energies as compared to a prediction of a one-dimensional potential model [1-5]. This is in marked contrast to high energy nuclear reactions, in which the couplings play a much less important role and thus the reaction dynamics is much simpler.

Secondly, fusion offers a unique opportunity to study quantum tunneling with many degrees of freedom. That is, fusion takes place only by quantum tunneling at energies below the Coulomb barrier, and the subbarrier enhancement of fusion cross sections can be viewed as a result of coupling assisted tunneling. Heavy-ion fusion reactions are unique in this respect because a variety of intrinsic degrees of freedom are involved, such as static and dynamical nuclear deformations with several multipolarities, as well as several types of particle transfer processes with several values of a transfer $Q$-value, which can be both negative and positive. Also, in heavy-ion fusion reactions, the incident energy can be easily varied in order to study the energy dependence of the tunneling probability, whereas the energy is basically fixed in many other tunneling phenomena in nuclear physics, such as alpha decays.

In order to analyze heavy-ion subbarrier fusion reactions, the coupled-channels approach has been developed $[1,6]$, which has enjoyed a lot of success in reproducing experimental data in many reaction systems. In this contribution, we shall first overview the present status of this approach and then discuss several remaining theoretical challenges.

\section{Coupled-channels approach}

The field of heavy-ion subbarrier fusion started at the late 70's, when a large subbarrier enhancement of fusion cross sections was discovered e.g., in the ${ }^{16} \mathrm{O}+{ }^{154} \mathrm{Sm}$ system [7]. For this particular system, the enhancement of fusion cross sections has been well understood in terms of the deformation of the target nucleus, ${ }^{154} \mathrm{Sm}$. This nucleus is a typical 
deformed nucleus with a quadrupole deformation parameter of $\beta_{2} \sim 0.3$. When the target nucleus is deformed, the potential between the projectile and the target nuclei depends upon the orientation angle, $\theta$, of the deformed target. The fusion cross sections are then computed as,

$$
\sigma_{\text {fus }}(E)=\int_{0}^{1} d(\cos \theta) \sigma_{\text {fus }}(E ; \theta),
$$

where $\sigma_{\text {fus }}(E ; \theta)$ is the fusion cross section at the incident energy $E$ for a fixed orientation angle $\theta$. This formula well reproduces experimental fusion cross sections for many systems with a deformed target nucleus, including the ${ }^{16} \mathrm{O}+{ }^{154} \mathrm{Sm}$ system $[1,8]$.

Eq. (1) is valid only when the excitation energy of a rotational excitation can be neglected as compared to the curvature of the Coulomb barrier [1]. In more general cases, one needs to solve the coupled-channels equations,

$$
\left[-\frac{\hbar^{2}}{2 \mu} \nabla^{2}+\epsilon_{k}-E\right] \psi_{k}(\boldsymbol{r})+\sum_{k^{\prime}}\left\langle\phi_{k}|V(\boldsymbol{r}, \xi)| \phi_{k^{\prime}}\right\rangle \psi_{k^{\prime}}(\boldsymbol{r})=0,
$$

where $\mu$ is the reduced mass for the relative motion between the colliding nuclei, $\phi_{k}$ is the intrinsic wave function, for which $\epsilon_{k}$ is the excitation energy. $V(r, \xi)$ is the total potential, which includes both the bare and the coupling potentials, $\xi$ denoting the intrinsic coordinate. Notice that the coupled-channels equations, Eq. (2), are derived by expanding the total wave function, $\Psi$, with the basis functions $\phi_{k}$, as,

$$
\Psi(\boldsymbol{r}, \xi)=\sum_{k} \psi_{k}(\boldsymbol{r}) \phi_{k}(\xi)
$$

Important ingredients for the coupled-channels approach are the internuclear potential, $V$, as well as the nature of the intrinsic degrees of freedom, $\xi$. For the latter, one often employs the macroscopic collective model [1]. Such approach has successfully accounted for experimental data for many systems, and has been a standard tool in analyzing experimental data for heavy-ion subbarrier fusion reactions [6]. The coupled-channels approach also offers a natural explanation for the barrier distribution [9], which is intimately related to the eigenchannel representation of the coupled-channels equations [1].

\section{Remaining challenges in the coupled-channels approach}

\subsection{Deep subbarrier hindrance of fusion cross sections}

Despite its success, there are still several theoretical challenges in the coupled-channels approach to subbarrier fusion. In this section, we discuss four main challenges.

The first problem which we consider is the hindrance phenomena of fusion cross sections at deep subbarrier energies [4]. That is, even when a standard coupled-channels calculation well reproduces experimental fusion cross sections in the vicinity of the Coulomb barrier, it appears that

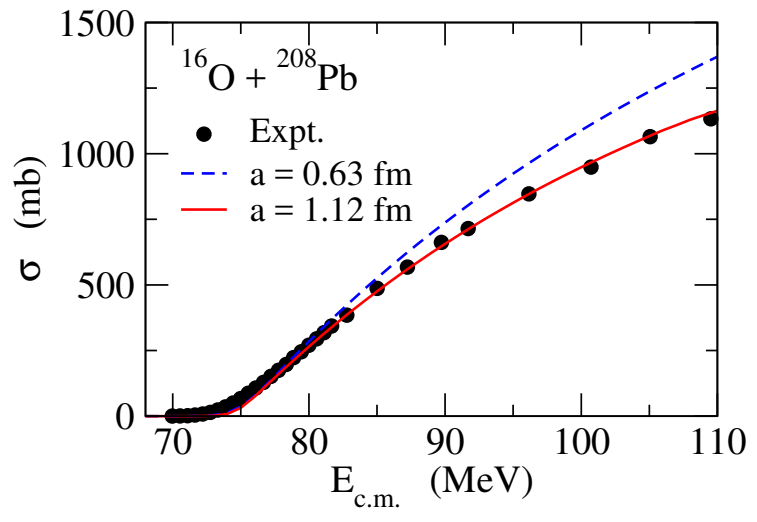

Figure 1. Fusion cross sections for the ${ }^{16} \mathrm{O}+{ }^{208} \mathrm{~Pb}$ system. The dashed and the solid lines denote the results of single-channel calculations with a Woods-Saxon internuclear potential with the surface diffuseness parameter of $a=0.63$ and $1.12 \mathrm{fm}$, respectively. The experimental data are taken from Ref. [15].

such calculation tends to overestimate fusion cross sections at deep subbarrier energies. Even though the exact origin for this phenomenon has not yet been clarified, so far there are mainly two models which account for the deep subbarrier hindrance of fusion cross sections. One is based on the sudden approach, in which fusion reaction is assumed to take place so fast that the density distribution of each colliding nucleus is frozen during fusion $[4,10]$. The frozen density approximation leads to a repulsive core in an internucleus potential, which results in a shallow pocket. High angular momenta are cut-off when a potential is shallow, and this angular momentum cut-off is the main cause of deep subbarrier hindrance in this model. The second model, on the other hand, is based on the adiabatic approach, in which reaction is assumed to take place so slowly that the density distribution is optimized at every instant $[11,12]$. This results in a deep and thick internucleus potential, and a tunneling of such thick potential has a responsibility to hinder fusion cross sections.

So far, both models have been equally successful in reproducing the observed fusion hindrance phenomenon. In order to disentangle them, it will be necessary to properly model the dynamics, such as the energy dissipation, around and after the touching point of the colliding nuclei [13], for which most of the current approaches resort either to the incoming wave boundary condition [1] or to a short range imaginary potential. Such modeling will be important also to understand the dynamics of quasi-fission and fusion reactions relevant to superheavy nuclei.

\subsection{Fusion above the barrier}

We next discuss fusion cross sections at energies above the Coulomb barrier. It has been a long standing problem that a Woods-Saxon internuclear potential with a standard value of surface diffuseness parameter, that is, $a$ $0.63 \mathrm{fm}$, systematically overestimates fusion cross sections above the Coulomb barrier [14]. If the surface diffuseness parameter is phenomenologically increased, experimental data appear to be reproduced (see Fig. 1 for a 


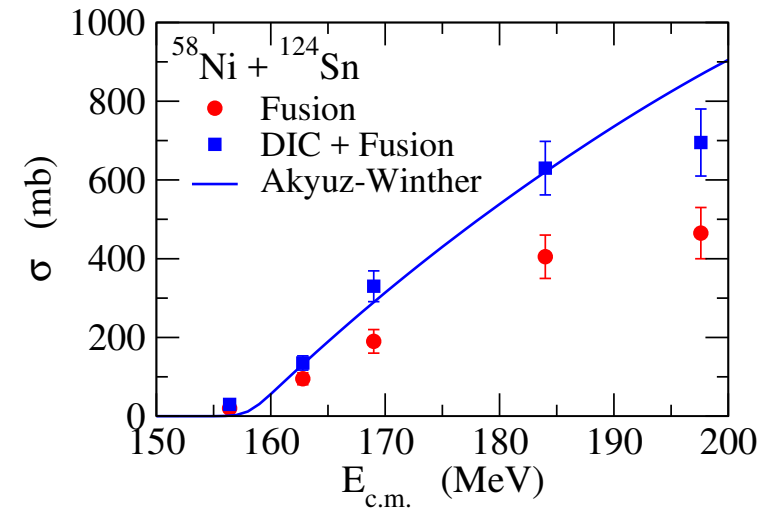

Figure 2. The experimental data for fusion cross sections (the filled circles) and a sum of fusion and deep-inelastic collision cross sections (the filled squares) for the ${ }^{58} \mathrm{Ni}+{ }^{124} \mathrm{Sn}$ system. These data are taken from Ref. [17]. The solid line shows the result of a single-channel calculation with the Akyüz-Winter potential [18].

typical example of the situation, that is, the fusion cross sections for the ${ }^{16} \mathrm{O}+{ }^{208} \mathrm{~Pb}$ system), but the exact origin of such surface diffuseness anomaly has not yet been understood.

The situation shown in Fig. 1 appears similar to the one in heavier systems where deep-inelastic collision well competes with fusion. In Ref. [16], Reisdorf argued that a barrier passing calculation well accounts for a sum of fusion and deep-inelastic cross sections e.g., for the ${ }^{58} \mathrm{Ni}+{ }^{124} \mathrm{Sn}$ system, even though it overestimates fusion cross sections themselves (see Fig. 2). The overestimate of fusion cross sections shown in Fig. 1 may have a similar origin [14].

It has been known that energy and angular momentum dissipations play an important role in deep inelastic collisions. Therefore, in order to resolve the surface diffuseness anomaly, it is crucially important to understand the nuclear dissipation in heavy-ion reactions. So far, current fusion models are all "friction free", in a sense that friction is taken into account only as a strong absorption well inside the barrier. It will be important to extend it by including the effect of dissipations at larger distances. Such model will provide a quantal model for deep inelastic collisions, and at the same time it will also describe dissipative tunneling in heavy-ion fusion reactions [19].

\subsection{Fusion of unstable nuclei}

Fusion of unstable nuclei has been discussed for some time [5, 20-22], but its underlying dynamics has not yet been completely understood. For unstable nuclei, the breakup of projectile nucleus becomes important because of its weakly bound property. This process complicates the whole fusion process, especially when one intends to separate between complete and incomplete fusion cross sections [5, 20]. Furthermore, transfer processes may also significantly affect the dynamics of fusion reactions of weakly bound nuclei [23-25]. It is therefore important for any theoretical calculation to take into account

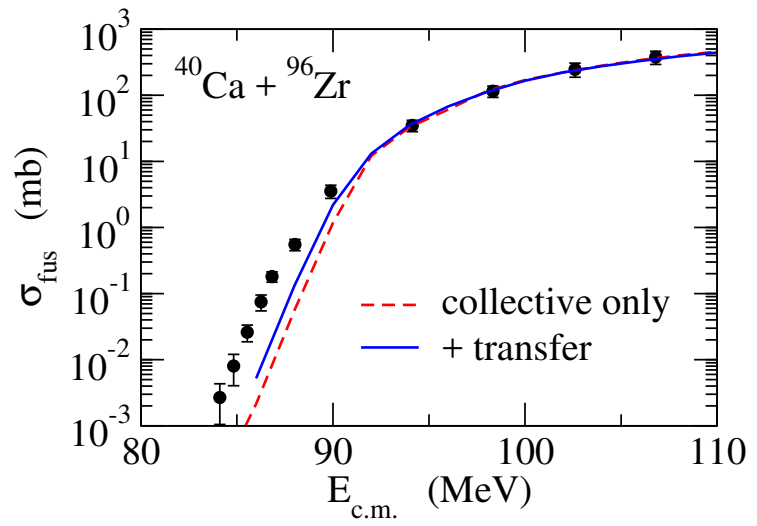

Figure 3. Fusion cross sections for the ${ }^{40} \mathrm{Ca}+{ }^{96} \mathrm{Zr}$ system. The dashed line is obtained by including only the collective octupole phonon excitations in the projectile and the target nuclei, while the solid line is obtained by including in addition the multineutron transfer channels. The strengths of the transfer couplings are determined so as to reproduce the experimental transfer cross sections for this system. The experimental data for fusion cross sections are taken from Ref. [34].

fusion, breakup, and transfer processes simultaneously in order to model fusion of unstable nuclei. Such calculation would require a large scale computing, and has been rather scarce. An exception is a time-dependent wave packet approach [26], which however has been limited only to total fusion cross sections. Because of this, the enhancement of fusion cross sections observed e.g., in the ${ }^{15} \mathrm{C}+{ }^{232} \mathrm{Th}$ system [27], as compared to fusion cross sections for the ${ }^{12,13,14} \mathrm{C}+{ }^{232} \mathrm{Th}$ systems, has remained theoretically unexplained.

\subsection{Interplay between fusion and transfer}

The nucleon transfer processes, especially the two-neutron transfer process, are important subjects not only in subbarrier fusion but also in connection to the pairing correlation in neutron-rich nuclei [28-30]. The multi-neutron transfer process [31] would play an important role also in fusion of neutron-rich skin nuclei, in order to reach the island of stability in the superhevy region.

An important theoretical issue here is to reproduce experimental fusion and transfer cross sections simultaneously. Recently, we have investigated this problem for the ${ }^{40} \mathrm{Ca}+{ }^{96} \mathrm{Zr}$ system [32]. By including the multi-neutron transfer process in the coupled-channels approach, we have found that the coupling strengths for the transfer couplings, which reproduce the transfer cross sections, largely underestimate fusion cross sections (see Fig. 3). To this end, we have included one octupole phonon excitation in ${ }^{40} \mathrm{Ca}$, the octupole phonon excitations in ${ }^{96} \mathrm{Zr}$ up to the three-phonon levels, and the multi-nucleon transfer process up to three-neutron transfer, with both simultaneous and direct two-neutron transfer couplings [32]. We have assumed a transfer to a single effective channel for each transfer partition, and set its energy to be the same as the optimum $Q$-value, $Q=0$. Following Ref. [33], we have assumed that the properties of the collective excitations do 


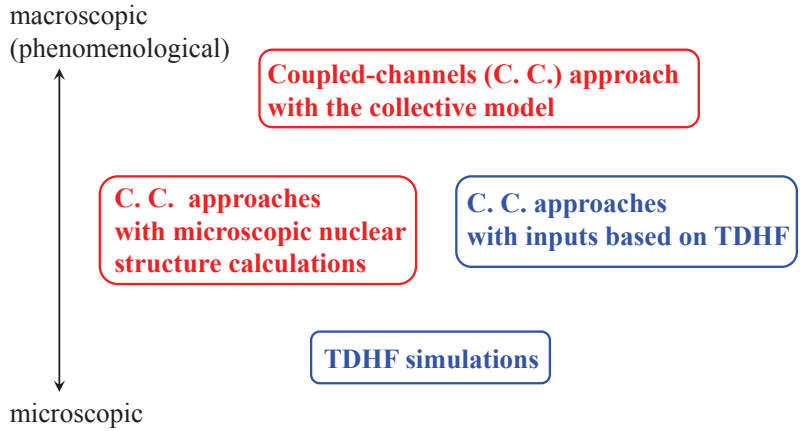

Figure 4. The current status of theoretical approaches to heavyion subbarrier fusion reactions.

not change even after the transfer. A striking fact is that the transfer process enhances fusion cross sections only a little if the coupling strengths are chosen so as to reproduce the transfer data. This indicates that the coupling scheme which has been employed in this calculation needs a further extension, by taking into account e.g., a distribution of transfer $Q$-value as well as changes in collective states after transfer. We have reached a similar conclusion also for the ${ }^{40} \mathrm{Ca}+{ }^{58,64} \mathrm{Ni}$ systems [35].

Very recently, it has been found experimentally that the multi-nucleon transfer processes in ${ }^{16,18} \mathrm{O},{ }^{19} \mathrm{~F}+{ }^{208} \mathrm{~Pb}$ reactions populate highly excited states in the target-like nuclei [36]. A population of high excited states would lead to energy dissipation. It would be another challenging problem to describe such process within the framework of coupled-channels approach [19].

\section{Semi-microscopic approach to heavy-ion fusion reactions}

\subsection{Current status of fusion modelings}

Figure 4 summarizes the current status of theoretical approaches to heavy-ion subbarrier fusion reactions. The coupled-channels approach with the collective model is categorized as a macroscopic and phenomenological approach, which needs empirical information on the coupling strengths and an internuclear potential. On the other hand, a full time-dependent Hartree-Fock (TDHF) simulation, which has rapidly been developed in the past few years [37-39], does not require any empirical input once the energy functional is specified. This is a microscopic approach, starting from the nucleon degree of freedom. A big challenge in this approach, however, is a well known fact that TDHF cannot describe quantum tunneling, and thus heavy-ion fusion reactions at energies below the Coulomb barrier. One would have to go beyond the meanfield approximation in order to resolve this problem, e.g., by using the time-dependent generator coordinate method (TDGCM) [40]. No realistic calculation, however, has been done so far because such calculation is still quite numerically expensive.

In between the macroscopic and the full microscopic approaches, one can also think about semi-microscopic approaches. These are coupled-channels calculations with inputs either from a microscopic nuclear structure calculation [41, 42] or from a TDHF simulation [43-45]. The density-constrained TDHF (DC-TDHF) method [43] is categorized into this group. These semi-microscopic approaches can be applied to fusion at energies below the Coulomb barrier, as one solves a quantum mechanical equation once it is set up using microscopic inputs.

\subsection{Coupled-channels calculations with microscopic nuclear structure calculations}

As an example of the semi-microscopic approaches to heavy-ion subbarrier fusion reactions, we present in this subsection the approach which combines the coupledchannels calculations with the multi-reference covariant density functional theory (MR-CDFT) for nuclear collective excitations [41]. In this approach, the nuclear potential in the coupled-channels equations, Eq. (2), is assumed to be a deformed Woods-Saxon type with a microscopic multipole operator, $Q_{\lambda \mu}=\sum_{i} r_{i}^{\lambda} Y_{\lambda \mu}\left(\hat{\boldsymbol{r}}_{i}\right)$. That is,

$$
V(r, \xi)=\frac{-V_{0}}{1+\exp \left(\frac{r-R_{0}-\sqrt{\frac{2 \lambda+1}{4 \pi}} R_{T} \alpha_{\lambda 0}}{a}\right)}
$$

with

$$
\alpha_{\lambda \mu}=\frac{4 \pi}{3 e} \frac{1}{Z_{T} R_{T}^{\lambda}} Q_{\lambda \mu}
$$

where $R_{T}$ and $Z_{T}$ are the radius and the atomic number of the target nucleus, respectively (we here consider excitations in the target nucleus). In writing Eq. (4), we have used the isocentrifugal approximation [1]. The Coulomb coupling is also taken into account in a similar fashion. The wave functions for the collective states, $\phi_{k}(\xi)=\phi_{I M}(\xi)$, in Eq. (3) are many-body wave functions generated from microscopic nuclear structure calculations, such as the multi-reference covariant density functional theory. These microscopic wave functions also yield all the matrix elements of the multipole operator, $\left\langle\varphi_{I 0}\left|Q_{\lambda 0}\right| \varphi_{I^{\prime} 0}\right\rangle$. The coupled-channels equations can then be constructed in a similar way as in the macroscopic approach for a given internuclear potential.

In heavy-ion fusion reactions, the coupling between the ground state and the lowest-lying collective state play the most important role, even though the couplings from the lowest-lying state to higher states are often important as well. Since the strength for the coupling between the ground state and the lowest-lying state can often be estimated from an experimental transition probability, we introduce an overall scaling factor to all the matrix elements so that the transition from the lowest-lying collective state to the ground state is consistent with experimental data. The MR-CDFT calculation then provides the relative strengths among collective levels, which are often not available experimentally. The excitation energy, on the other hand, is often known well for many levels, and we simply use them in the calculations whenever they are available. 


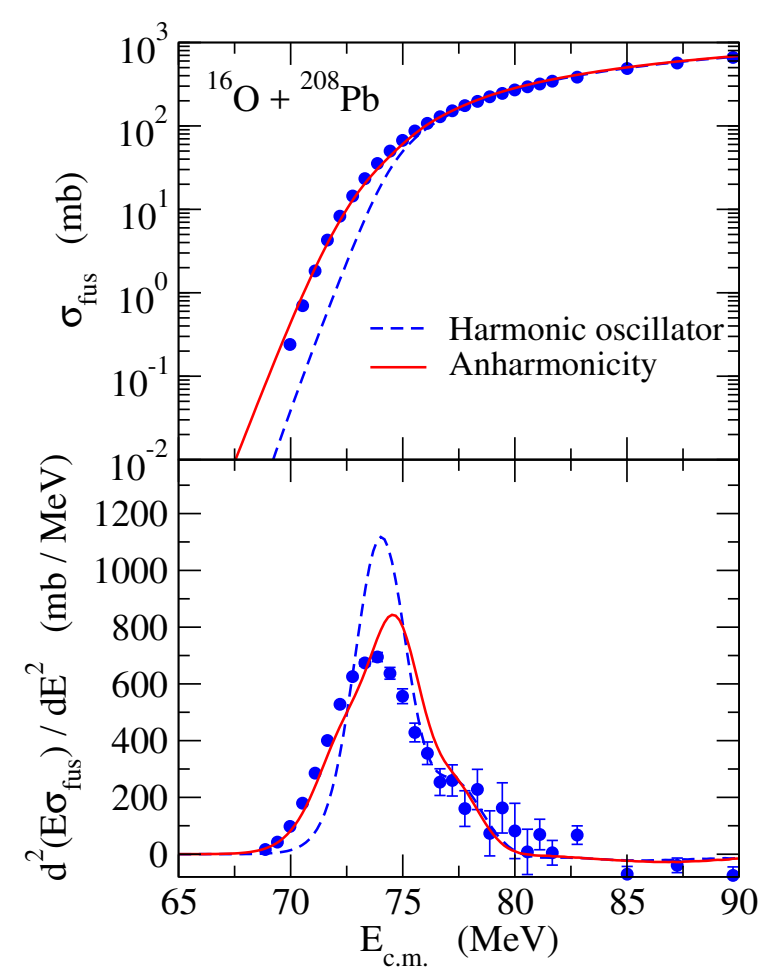

Figure 5. The fusion cross sections (upper panel) and the fusion barrier distribution (lower panel) for the ${ }^{16} \mathrm{O}+{ }^{208} \mathrm{~Pb}$ system obtained with the semi-microscopic coupled-channels calculation (the solid line). For this purpose, the multi-reference covariant density functional theory is employed, and states up to "two-phonon" configurations are included in the calculation. The dashed line shows the results of the two-phonon coupling in the harmonic oscillator limit. The experimental data are taken from Ref. [15].

Figures 5 shows the fusion cross section $\sigma_{\text {fus }}(E)$ and the fusion barrier distribution $D_{\text {fus }}(E)=d^{2}\left(E \sigma_{\text {fus }}\right) / d E^{2}$ $[2,9]$ for the ${ }^{16} \mathrm{O}+{ }^{208} \mathrm{~Pb}$ reaction so obtained. The dashed line shows the result of the coupled-channels calculations including up to the double quadrupole and octupole phonon states (that is, $3_{1}^{-}, 2_{1}^{+},\left(3_{1}^{-}\right)^{2},\left(2_{1}^{+}\right)^{2}$, and $3_{1}^{-} \otimes 2_{1}^{+}$ states) in ${ }^{208} \mathrm{~Pb}$ in the harmonic oscillator limit. As shown in the figure, this calculation overestimates the height of the main peak in the barrier distribution, as in many previous calculations $[15,46,47]$. The result of the semimicroscopic coupled-channels calculations is shown by the solid line. For this purpose, we generate the collective states with the MR-CDFT approach with the PC-PK1 interaction [48]. In the coupled-channels calculation, in addition to the entrance channel, we include the one-octupole phonon state, $3_{1}^{-}$, at $2.615 \mathrm{MeV}$, the "one-quadrupole" phonon state, $2_{1}^{+}$, and several states which are strongly coupled to those $3_{1}^{-}$and $2_{1}^{+}$states by the octupole and the quadrupole couplings. The whole two-octupole-phonon candidate states are included in this model space. It is remarkable that the semi-microscopic calculation yields a much lower main peak in the fusion barrier distribution, and the agreement with the experimental data is considerably improved both for the fusion cross sections and for the barrier distribution.
For this good reproduction, we find that the coupling between the $3_{1}^{-}$and the $2_{1}^{+}$states play an important role. The couplings between the two-octupole-phonon states and the excited negative parity states also play a role. In the calculations in the harmonic oscillator limit, the $3^{-}$, $2_{1}^{+}$and the $5_{1}^{-}$states are treated as independent phonon states, and the couplings among those states are absent. In contrast, in the present semi-microscopic calculation, the $2_{1}^{+}$state has in part the two octupole phonon character, $\left(3^{-}\right)^{2}$. Likewise, the $1^{-}$and $5_{1}^{-}$states have both the $\left(3^{-}\right)^{3}$ and the $3^{-} \otimes 2^{+}$characters. Apparently those anharmonicity effects in the transition strengths lead to the strong couplings between the ground state and those states via multiple octupole excitations, significantly improving the previous coupled-channels calculations.

\section{Summary}

Heavy-ion subbarrier fusion reactions show a strong interplay between nuclear reaction and nuclear structure, and contain a variety of rich physics, such as coupling assisted tunneling and energy dissipation. The coupled-channels approach with a macroscopic and phenomenological description for nuclear structure has been developed in order to understand the dynamics of subbarier fusion. We have argued in this paper that a theoretical description of subbarrier fusion is now gradually being shifted from the phenomenological approach to more microscopic modelings. A full time-dependent Hartree-Fock simulation is a good example for this, even though this approach still has a difficulty in applying to fusion at energies below the Coulomb barrier. We have also developed the semimicroscopic coupled-channels approach, which uses inputs generated from microscopic nuclear structure calculations. We have shown the result of such calculation for the ${ }^{16} \mathrm{O}+{ }^{208} \mathrm{~Pb}$ system, which has considerably improved the previous coupled-channels calculations in the harmonic oscillator limit. This approach is more flexible than the conventional approach for the coupled-channels calculations, because it can be applied also to transitional nuclei, which show neither the vibrational nor the rotational characters.

There are still many theoretical challenges in heavyion subbarrier fusion reactions, for which dissipation and multi-nucleon transfer make two important keywords. These are: i) deep subbarrier hindrance of fusion cross sections, ii) fusion above the barrier and the role of nuclear dissipation, iii) fusion of unstable nuclei, and iv) an interplay between fusion and transfer. Microscopic and semimicroscopic approaches to subbarrier fusion will hopefully resolve some of these challenges in a near future.

We thank J.M. Yao and G. Scamps for collaborations and for useful discussions.

\section{References}

[1] K. Hagino and N. Takigawa, Prog. Theor. Phys. 128, 1061 (2012). 
[2] M. Dasgupta, D.J. Hinde, N. Rowley and A.M. Stefanini, Annu. Rev. Nucl. Part. Sci. 48, 401 (1998).

[3] A.B. Balantekin and N. Takigawa, Rev. Mod. Phys. 70, 77(1998).

[4] B.B. Back, H. Esbensen, C.L. Jiang and K.E. Rehm, Rev. Mod. Phys. 86, 317 (2014), and references therein.

[5] L.F. Canto, P.R.S. Gomes, R. Donangelo, J. Lubian and M.S. Hussein, Phys. Rep. 596, 1 (2015).

[6] K. Hagino, N. Rowley, and A.T. Kruppa, Comp. Phys. Comm. 123, 143 (1999).

[7] R.G. Stokstad et al., Phys. Rev. Lett. 41, 465 (1978).

[8] J.R. Leigh et al., Phys. Rev. C52, 3151 (1995).

[9] N. Rowley, G.R. Satchler, and P.H. Stelson, Phys. Lett. B254, 25 (1991).

[10] S. Misicu and H. Esbensen, Phys. Rev. Lett. 96, 112701 (2006).

[11] T. Ichikawa, K. Hagino, and A. Iwamoto, Phys. Rev. Lett. 103, 202701 (2009).

[12] T. Ichikawa, Phys. Rev. C92, 064604 (2015).

[13] T. Ichikawa, K. Hagino, and A. Iwamoto, Phys. Rev. C75, 064612 (2007).

[14] J.O. Newton et al., Phys. Lett. B586, 219 (2004); Phys. Rev. C70, 024605 (2004).

[15] C.R. Morton et al., Phys. Rev. C60, 44608 (1999).

[16] W. Reisdorf, J. of Phys. G20, 1297 (1994).

[17] F.L.H. Wolfs, Phys. Rev. C36, 1379 (1987).

[18] O. Akyüz and A. Winther, Proc. Int. School of Physics Enrico Fermi, Course LXXVII, ed. by R.A. Broglia, R.A. Ricci, and C.H. Dasso (North-Holland, Amsterdam, 1981).

[19] M. Tokieda and K. Hagino, arXiv:1704.00254.

[20] L.F. Canto, P.R.S. Gomes, R. Donangelo, and M.S. Hussein, Phys. Rep. 424, 1 (2006).

[21] K. Hagino, A. Vitturi, C.H. Dasso, and S.M. Lenzi, Phys. Rev. C61, 037602 (2000).

[22] A. Diaz-Torres and I. Thompson, Phys. Rev. C65, 024606 (2002).

[23] R. Raabe et al., Nature 431, 823 (2004).

[24] A. Lemasson et al., Phys. Rev. Lett. 103, 232701 (2009).

[25] D.H. Luong et al., Phys. Rev. C88, 034609 (2013).
[26] M. Ito, K. Yabana, T. Nakatsukasa, and M. Ueda, Phys. Lett. B637, 53 (2006).

[27] M. Alcorta et al., Phys. Rev. Lett. 106, 172701 (2011).

[28] W. von Oertzen and A. Vitturi, Rep. Prog. Phys. 64, 1247 (2001).

[29] K. Hagino and H. Sagawa, Phys. Rev. C72, 044321 (2005).

[30] G. Potel et al., Phys. Rev. Lett. 107, 092501 (2011).

[31] L. Corradi, G. Pollarolo, and S. Szilner, J. of Phys. G36, 113101 (2009).

[32] G. Scamps and K. Hagino, Phys. Rev. C92, 054614 (2015).

[33] H. Esbensen and S. Landowne, Nucl. Phys. A492, 473 (1989).

[34] A.M. Stefanini et al., Phys. Lett. B728, 639 (2014).

[35] G. Scamps, D. Bourgin, K. Hagino, F. Haas, and S. Courtin, arXiv:1609.06428.

[36] D.C. Rafferty et al., Phys. Rev. C94, 024607 (2016).

[37] C. Simenel, Euro. Phys. J. A48, 152 (2011).

[38] K. Sekizawa and K. Yabana, Phys. Rev. C88, 014614 (2013); C93, 054616 (2016).

[39] G. Scamps and D. Lacroix, Phys. Rev. C87, 014605 (2012).

[40] P.-G. Reinhard, R.Y. Cusson, and K. Goeke, Nucl. Phys. A398, 141 (1983).

[41] K. Hagino and J.M. Yao, Phys. Rev. C91, 064606 (2015); J.M. Yao and K. Hagino, Phys. Rev. C94, 011303(R) (2016).

[42] T. Ichikawa and K. Matsuyanagi, Phys. Rev. C88, 011602(R) (2013); Phys. Rev. C92, 021602(R) (2015).

[43] A.S. Umar and V.E. Oberacker, Phys. Rev. C77, 064605 (2008); Euro. Phys. J. A39, 243 (2009).

[44] K. Washiyama and D. Lacroix, Phys. Rev. C78, 024610 (2008); Phys. Rev. C79, 024609 (2009).

[45] C. Simenel, M. Dasgupta, D.J. Hinde, and E. Williams, Phys. Rev. C88, 064604 (2013).

[46] H. Esbensen and S. Misicu, Phys. Rev. C 76, 054609 (2007).

[47] S. Yusa, K. Hagino, and N. Rowley, Phys. Rev. C85, 054601 (2012).

[48] P.W. Zhao et al., Phys. Rev. C82, 054319 (2010). 